\title{
Transport Et Approvisionnement Des Produits Vivriers A Abidjan En Côte d'Ivoire
}

\author{
Kouassi Mangoua Akissi Hélène-Francette \\ Doctorant, Université Félix Houphouet-Boigny, \\ Cocody-Abidjan, Côte d'Ivoire \\ Koffi Lath Franck Eric
}

Maître-Assistant, Université Peleforo Gon Coulibaly de Korhogo,

Côte d'Ivoire

Aloko N'guessan Jérôme

Directeur de recherches, Université Félix Houphouet-Boigny,

Cocody-Abidjan, Côte d'Ivoire

doi: 10.19044/esj.2017.v13n26p212 URL:http://dx.doi.org/10.19044/esj.2017.v13n26p212

\begin{abstract}
The population of Abidjan is growing rapidly. This demographic mass creates a food needs. To answer this demand, transport service is essential to reach the production areas. The contribution explains an overview of the transport service in a supply chain of food to Abidjan. The methodology research is based on two facts: the bibliographic research and field surveys. The study shows the meaning of transport which may vary according to the scales and levels of supply. Difficult conditions to access production sites, road traffic harassment and degradation of road infrastructure significant are problems faced by transporters in carrying out their activities.
\end{abstract}

Keywords: Côte d'Ivoire, Abidjan, transport, supply, farm products, markets

\section{Résumé}

La population d'Abidjan connaît un accroissement rapide. Cette masse démographique crée des besoins alimentaires accrus. Pour répondre à la demande, le recours au service de transport pour se rendre dans les zones de production de plus en plus éloignées s'avère indispensable. Cette contribution propose un schéma explicatif général du rôle que joue le transport dans l'approvisionnement d'Abidjan en produits vivriers. La méthodologie de la recherche repose sur deux piliers : une recherche bibliographique et des enquêtes de terrain. Il ressort de cette étude que les moyens de transport utilisés varient selon les échelles et les niveaux 
d'approvisionnement. Les conditions d'accès difficiles aux lieux de production, les tracasseries routières et la dégradation des infrastructures routières sont autant de contraintes auxquelles font face les transporteurs dans l'exercice de leurs activités.

Mots-clés : Côte d'Ivoire, Abidjan, transport, approvisionnement, produits vivriers, marchés

\section{Introduction}

Le secteur des transports est fondamental dans la vie de nos sociétés où chacun se déplace continuellement, où la plupart des produits consommés viennent d'ailleurs et où circulent continuellement l'argent, les images, les informations, les hommes et les produits (Mérenne, 2013). La vie économique et sociale n'aurait aucune possibilité de s'exercer sans les transports. Le transport constitue donc un élément essentiel dans tous les domaines de l'activité quotidienne et est déterminant à l'exigence alimentaire croissante des villes, notamment Abidjan.

Abidjan connait une croissance démographique spectaculaire. Elle abrite à elle seule $20 \%$ de la population totale de la Côte d'Ivoire et $60 \%$ de la population urbaine du pays (INS, 2014). Cette croissance s'accompagne d'une urbanisation accélérée difficilement maitrisable. L'explosion démographique urbaine se traduit par une explosion concomitante de la demande de produits alimentaires de base. Cette demande croissante alimentaire est satisfaite à partir des zones rurales de plus en plus lointaines ainsi que par un arrière-pays agricole étendu. Ainsi, l'approvisionnement rapide, régulier et en quantités suffisantes est donc un impératif. Cela suppose un accès plus aisé aux zones et nécessite un système de transport performant, adapté, efficace, régulier, réglementé et surtout bien organisé, condition sine qua non pour l'écoulement des produits.

Cependant, le transfert des produits vivriers du lieu de production vers les zones de consommation pose d'énormes problèmes. L'objectif poursuivi par cette contribution propose un schéma explicatif général du rôle que joue le transport dans l'approvisionnement d'Abidjan en produits vivriers.

\section{Methodologie}

La méthodologie repose sur une recherche bibliographique et des enquêtes de terrain.

Concernant la recherche bibliographique, les informations collectées ont permis d'appréhender la manière dont ces produits sont transportés depuis les bassins de production jusqu'aux marchés d'Abidjan. Elle a permis également de collecter des données statistiques démographiques et de 
transport. Les données démographiques sont issues des différents RGPH $(1975,1988,1998$ et 2013) et des estimations de 2014 de l'institut National de la Statistique (INS) de Côte d'Ivoire. Quant aux données relatives au transport, elles proviennent de la base de données du ministère du transport, de 1 'AGEROUTE et du Fond d'Entretien Routier (FER).

Concernant les enquêtes de terrain, deux catégories d'acteurs ont été interrogées à savoir, les transporteurs et les grossistes.

La détermination des différents échantillons s'est faite à partir de la formule de Fisher suivante :

$\mathbf{n}=t^{2} \frac{p(1-p) x^{2}}{m^{2}}$ avec

$\mathrm{n}=$ taille d'échantillon requise

$\mathrm{t}=$ niveau de confiance

$\mathrm{P}=$ proportion de la population cible

$\mathrm{m}=$ marge d'erreur

Le transport de vivrier est assuré par les particuliers. Ainsi, sur un effectif total de 191 transporteurs des produits, 144 (soit 75,39\%) repartis selon les communes ont été interrogés. Les transporteurs enquêtés ont été sélectionnés sur la base du sondage stratifié de la méthode aléatoire dont les communes ont constitué les strates. Quant aux grossistes, sur 614 grossistes dénombrés par l'OCPV, 153 grossistes ont été interrogés. La marge d'erreur retenue est de 5\%.Aux transporteurs, il s'est agit de poser des questions sur les moyens de transport utilisés pour la marchandise, l'état des voies d'accès au zone de production, le prix du transport et les difficultés rencontrées dans l'écoulement des produits vivriers.

Pour les grossistes, il s'est agit de poser des questions sur les bassins d'approvisionnement, le mode d'achat effectué (gros, demi- gros ou en association avec d'autres vendeuses) et enfin le type de rapport entretenu avec les coopératives

L'application de cette méthodologie a abouti aux résultats suivants :

\section{Resultats et discussion}

\section{Les infrastructures de transport : etat des lieux} fer.

Il concerne la qualité et la quantité du réseau routier et du chemin de

\section{Réseau routier ivoirien}

La loi n83-788 du 2 Août 1983 établit quatre types de réseaux routiers. D'abord, nous avons les routes internationales et régionales (Classe A) d'une longueur de $7526 \mathrm{~km}$ dont $4513 \mathrm{~km}$ sont bitumées. Ensuite, nous avons les routes départementales (Classe B) d'une longueur d'environ 12526 $\mathrm{km}$ dont $1747 \mathrm{~km}$ sont bitumées. Enfin, nous avons les routes de sous- 
préfectures et de villages (Classe C) d'une longueur d'environ $34269 \mathrm{~km}$ dont $125 \mathrm{~km}$ sont bitumées. Au niveau quantitatif, le tableau ci-dessous nous signale que le réseau routier est passé de $24300 \mathrm{~km}$ en 1960 à $82000 \mathrm{~km}$ en 2015 , toutes catégories de routes confondues.

Tableau 1- Evolution du linéaire (en km) du réseau routier de 1960 à 2015

\begin{tabular}{|c|c|c|c|c|c|c|c|c|c|}
\hline Année & $\mathbf{1 9 6 0}$ & $\mathbf{1 9 7 0}$ & $\mathbf{1 9 8 0}$ & $\mathbf{1 9 8 5}$ & $\mathbf{1 9 9 0}$ & $\mathbf{1 9 9 5}$ & $\mathbf{2 0 0 0}$ & $\mathbf{2 0 1 0}$ & $\mathbf{2 0 1 5}$ \\
\hline $\begin{array}{c}\text { Routes en } \\
\text { terres } \\
(\mathbf{k m})\end{array}$ & 24300 & 33800 & 42300 & 49650 & 63300 & 62241 & 75000 & 75000 & 75500 \\
\hline $\begin{array}{c}\text { Routes } \\
\text { revêtues (km) }\end{array}$ & 700 & 1300 & 3100 & 3850 & 4700 & 5800 & 5000 & 4500 & 6500 \\
\hline Total & $\mathbf{2 5 0 0 0}$ & $\mathbf{3 5 0 0 0}$ & $\mathbf{4 5 4 0 0}$ & $\mathbf{5 3 0 0 0}$ & $\mathbf{6 8 0 0 0}$ & $\mathbf{6 8 0 4 1}$ & $\mathbf{8 0 0 0 0}$ & $\mathbf{7 0 5 0 0}$ & $\mathbf{8 2 0 0 0}$ \\
\hline
\end{tabular}

Source : Ageroute, 2015

L'analyse du tableau ci-dessus montre qu'entre 1960 et 1990, l'effort de réalisations routières a porté sur l'extension des routes en terres $(24300$ $\mathrm{km}$ à $75500 \mathrm{~km})$ et sur le développement du réseau revêtu $(700 \mathrm{~km}$ à 6500 $\mathrm{km})$. Cette situation place la Côte d'Ivoire dans le peloton de tête des pays africains. Deux éléments explicatifs pourraient nourrir la réflexion sur l'importance accordée à la route par les autorités ivoiriennes. Le premier élément se rapporte à l'économie qui repose sur l'agriculture génératrice de revenu. Le second élément est relatif au désenclavement des zones difficilement accessible en vue de faciliter les échanges nationaux et interétats pour amorcer son développement.

Cependant la qualité du réseau laisse indésirable malgré le linéaire important.

Tableau 2 - Réseau routier revêtu

\begin{tabular}{|c|c|c|c|c|c|c|}
\hline & Linéaire & \multicolumn{4}{|c|}{ Etat du réseau } \\
\cline { 3 - 7 } & total & $\begin{array}{c}\text { Très } \\
\text { bon }\end{array}$ & Bon & Moyen & Mauvais & $\begin{array}{c}\text { Très } \\
\text { mauvais }\end{array}$ \\
\hline Linéaire & 6500 & 599 & 1264 & 2472 & 763 & 1402 \\
\hline pourcentage & $100 \%$ & $9,21 \%$ & $19,4 \%$ & $38,1 \%$ & $11,7 \%$ & $21,6 \%$ \\
\hline
\end{tabular}

Source : Fond Entretien Routier, 2015

A la lecture du tableau 2, on constate un taux important de routes dégradées. L'état de ce réseau montre que près du tiers du réseau revêtu nécessite une intervention urgente bien qu'il y ait eu des avancées en termes de réhabilitations ces cinq dernières années. Les infrastructures routières qui, pendant longtemps ont été un avantage pour le commerce national et international de ce pays sont en passe de devenir une entrave pour le transport de marchandises. Le manque d'entretien régulier influe sur la qualité du service et ne permet pas une bonne circulation des biens et des 
personnes (Kassi, 2011). Face à une situation similaire, Djogo (1994) s'est rendu compte qu'au plan de la qualité, de l'efficacité et de la fiabilité, les infrastructures et services de transport sont sous-développés. Par ailleurs, la plupart des infrastructures routières est peu préservée, souffre d'inattention et est mal entretenue. En France, une étude menée par Cruz (2011) souligne que la route est le premier mode de transport avec $77 \%$ de $\mathrm{km}$ de routes.

En effet, la comparaison du réseau routier ivoirien $(82000 \mathrm{~km}$, dont $75500 \mathrm{~km}$ de routes en terre, $6500 \mathrm{~km}$ de routes bitumées, et $150 \mathrm{~km}$ d'autoroutes), au réseau routier français qui regroupe près de $950000 \mathrm{~km}$ de voies diverses et celui du Québec qui fait environ $185000 \mathrm{~km}$ d'autoroutes confirme que la route ivoirienne est "malade', Cependant la qualité peu reluisante des infrastructures routières n'est pas propre à la Côte d'Ivoire. Au Cameroun, Fofiri et al. (2011) ont prouvé que les routes bitumées représentent $10 \%$ du réseau. Vu le niveau de dégradation, la différence est peu perceptible entre routes dites en terre et celles rurales. En outre, les statistiques de la (CEA, 2007) sur les principaux indicateurs du réseau routier montrent que la route est le mode de transport dominant en Afrique subsaharienne ne présentent que $24,56 \%$ de routes revêtues caractérisées par la faiblesse de la densité de son réseau d'infrastructures de transport.

\section{L’unique voie ferrée}

Quantitativement, le réseau du chemin de fer manque d'une double ligne sur le tronçon Abidjan - Ouagadougou. De plus, il manque de matériel roulant surtout pour assurer le transport du vivrier notamment en zone urbaine auquel vient se greffer l'absence de nouveaux projets intérieurs. La qualité de service n'est pas de mise surtout du fait de la concurrence rail route qui tourne aujourd'hui en faveur de la route car le transport de personne par car et des produits vivriers par la route a énormément progressé depuis les années 1980. Le vieillissement des installations participe à la baisse de la qualité de service.

\section{Rôle des acteurs et organisation du transport Le rôle des acteurs}

Il existe deux catégories d'acteurs à savoir, les actifs et les semiactifs. Les actifs sont composés de transporteurs approvisionneurs, de coxer et de syndicats des transporteurs qui participent directement aux opérations de transport des produits vivriers. Les chauffeurs qui dominent la catégorie des actifs, transportent les produits bord champ à Abidjan. Quant aux coxer, ils font connaître les besoins de transport (capacité des camions, destinations) des demandeurs (commerçants ou producteurs) et les prix d'appel du transport proposés selon les destinations moyennant une commission. Le syndicat sert d'intermédiaire entre ses membres et les 
commerçants. Il a pour rôle le maintien de l'ordre et la répartition de façon équitable le travail entre les chauffeurs. Mais il agit également comme affréteur.

Les semi-actifs sont composés de commerçants transporteurs, de pseudo-transporteurs, de mécaniciens et d'axillaires de transport. Les commerçants-transporteurs (propriétaires) disposent d'un parc auto assez important et l'activité principale est exclusivement basée sur le commerce et le transport. Ces semi-actifs disposent d'une autorisation de transport et d'un nombre assez important de véhicule qu'ils mettent à la disposition des chauffeurs employés. Quant aux pseudo-transporteurs, ils disposent d'un ou de deux véhicules où il est très souvent le conducteur d'un véhicule. Le mécanicien vérifie l'état du véhicule relatif à la maintenance et à la réparation avant et après chaque voyage afin de minimiser les défaillances techniques. Les auxiliaires du transport, assure le transport des produits vers les autres marchés urbains. Ce résultat corrobore celui d'Echui (1993) qui, dans son étude identifie quatre principaux acteurs qui animent le transport des produits et assurent également la fonction d'affréteurs. A contrario, l'étude de Mpanzu (2012) menée au Congo met en jeu sept acteurs qui, sont le fruit d'une longue période d'adaptation de la filière dû aux multiples contraintes qui ont jalonné son parcours couronné de la défaillance de l'état à organiser le système de transport.

\section{Organisation du transport}

\section{Les formes d'organisation du transport des marchandises}

Trois formes d'organisations sont identifiées. La première forme d'organisation du transport est locale et se déroule à petite échelle. En effet, le transport fait objet de contact et de négociation direct entre le transporteur, le paysan ou le commerçant sans intermédiaire ni contrôle et intervention de la section locale du syndicat des transporteurs. Quant à la deuxième forme d'organisation, elle est régionale et obéit obligatoirement à la règle d'alignement appelé «tour de rôle » sous contrôle de la section locale du syndicat des transporteurs. La troisième forme d'organisation est terminale et a lieu à Abidjan.

Le transport s'organise aux abords des marchés et sur des aires de stationnement non aménagées le long de la voie de circulation. Pour la recherche de chargement, trois possibilités se présentent aux transporteurs. La première possibilité est basée sur les relations amicales et parentales à travers leurs propres démarches ou par les services des coxers avec qui des contrats sont passés. La deuxième possibilité s'offre après livraison dans un grand centre autre qu'Abidjan. Pour ne pas revenir vide, le chauffeur recherche dans la région du chargement retour à travers les commerçants en attente d'un moyen de transport le long de la route asphaltée. Cette pratique 
évite l'immobilisation du véhicule et les pertes de temps dues aux longues tournées de collecte. Quant à la troisième possibilité, le chauffeur traite avec le syndicat des transporteurs qui reçoit par l'intermédiaire des sections locales, des demandes de transport des commerçants. Ces sections locales négocient les prix et les conditions de transport selon les destinations moyennant une contrepartie financière. Cette contrepartie est de $10 \%$ sur le prix du transport pour le chargement d'un camion de capacité moyenne de 10 à 20 tonnes et de $25 \%$ sur le prix du transport s'il s'agit d'un ensemble articulé. Mais, les transporteurs sont soumis à des conditions de sélection afin d'éviter les pannes fréquentes qui entrainent de longue immobilisation durant le transport compte tenu de l'état des routes. Le critère de sélection est relatif à l'état du véhicule et à l'année d'acquisition. La quatrième possibilité obéît au système de transport des produits par contrat entre deux parties (les commerçants transporteurs et les coopératives) qu'ils sont tenues de le respecter. La coopérative s'en charge régulièrement du paiement de tous les frais d'entretien du parc auto du commerçant-transporteur. En contrepartie, ceux-ci s'engagent par contrat à transporter annuellement une quantité de sa marchandise fixée à l'avance.

Ces résultats sont en désaccord avec celui d'Echui(1993) qui retrace deux formes d'organisation. La forme structurée avec des cadres et agents de maîtrise qui s'occupent de la direction, de la comptabilité, de l'exploitation du parc et de la recherche du fret. La forme artisanale composée de personnels analphabètes assure la fonction de fret. A Kara au Togo, une étude menée par Simmala (2009) a conclu que le transport s'organise essentiellement au niveau de la gare routière. Il montre également que l'un des facteurs essentiels à prendre en compte dans cette organisation est le système du " tour de rôle »qui consiste à charger les véhicules selon leur ordre d'arrivée au point d'embarquement.

\section{Les moyens de transport utilisés}

Plusieurs types de moyens de transport sont utilisés en fonction de la distance, de la qualité du niveau de service routier et de l'importance des produits transportés. Par conséquent, la voiture est le moyen de transport dominant. Par contre, pour les moyens de transport non mécanisés, nous avons la marche avec portage sur la tête et à dos, les pousse-pousse et le tricycle comme le montre les photos 3,4 et 5 ci-dessous. Ces moyens de transport sont généralement utilisés sur de courtes distances pour transporter les petites quantités de peu d'importance. Tandis que le transport des produits sur la moyenne distance fait intervenir le transport mécanisé dont la charge utile n'excède pas plus d'une tonne (cf photo 2). Cependant, le transport des produits sur de longue distance se fait pour une large partie par les moyens mécanisés de grand gabarit comme le montre la photol ci- 
dessous. La figure 4 ci-après récapitule les moyens de transport utilisés dans le transfert des produits en fonction du niveau de service routier.

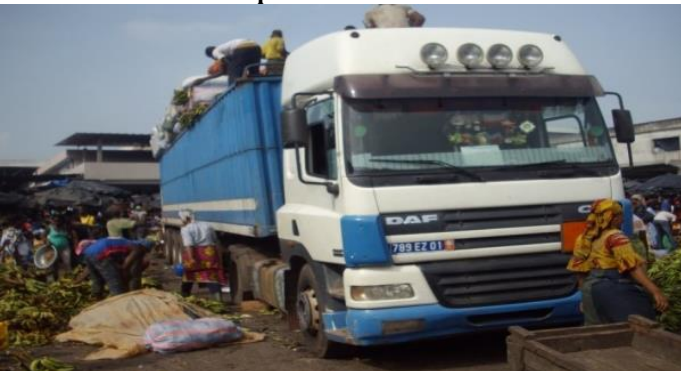

Photo 1. camion remorque (30 tonnes) provenant de Bouaflé ploie sous le poids de la banane plantain en plein déchargement au marché la Nouvelle Cocoprovi d'Adjamé. Le véhicule de grand gabarit est plus utilisé pour le transport des produits sur la longue distance. Cliché : Kouassi, 2017

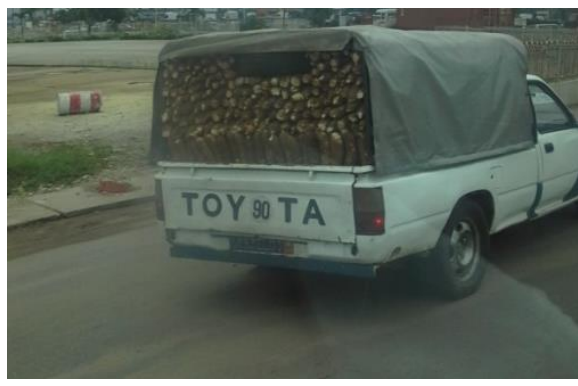

Photo 2. axe Bonoua-Abidjan, la bâchée pleine de manioc en partance pour Abidjan. Cette catégorie de véhicule est plus utilisée pour le transport des produits sur la moyenne distance. Cliché : Kouassi, 2017

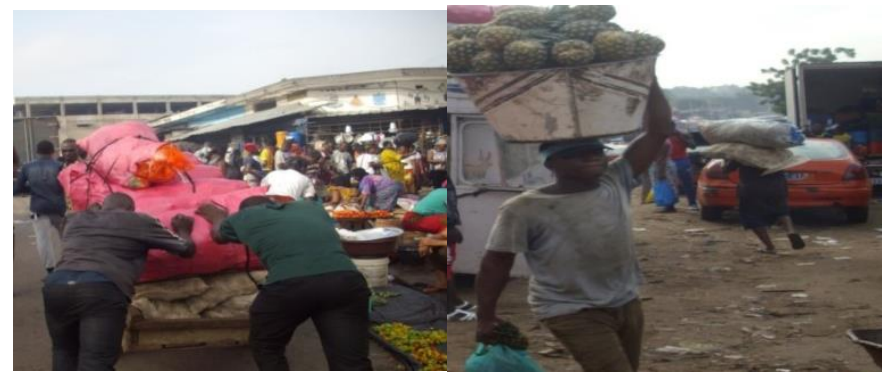

Photo 3. Une force motrice est exercée par les pousse-pousseurs sur le wotro dans le transport de produits

Cliché, Kouassi, 2017 sur la tête et à dos Cliché, Kouassi, 2017

Photo 4. la marche avec portage

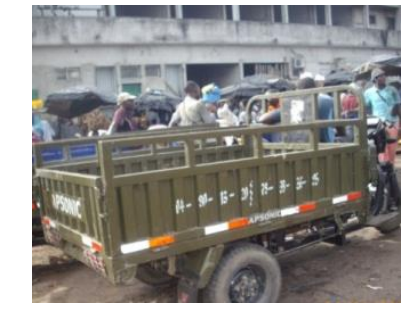

Photo 5. tricycle ou trois roues. Cliché : Kouassi, 2017

\section{Figure 1. Moyens de transport utilisés selon la qualité du niveau de service routier}

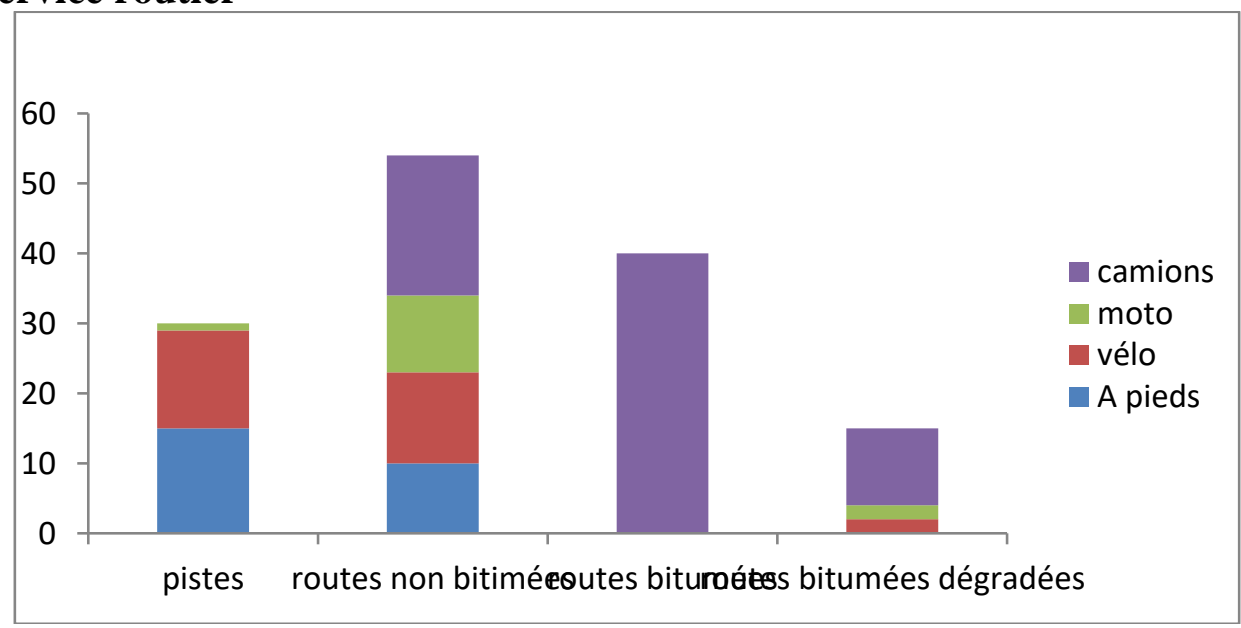

Source : Nos enquêtes, 2016 
Tableau 3. Types d'automobiles utilisées selon la distance et le volume de produits

\begin{tabular}{|c|c|c|}
\hline Distance parcourue en km & Type de véhicule & Capacité de charge \\
\hline Moins de 10 & $\begin{array}{c}\text { Marche à pied / } \\
\text { Bicyclette/tricycle }\end{array}$ & 200 à $800 \mathrm{~kg}$ \\
\hline 10 à 50 & $\begin{array}{c}\text { Mobylette/ Tricycle/ } \\
\text { Car de transport en commun }\end{array}$ & 3 tonnes \\
\hline $50-125$ & Bâché/frigo nette & $5-10$ tonnes \\
\hline $200-288$ & $\begin{array}{c}\text { Camionnette (pickup)/semi- } \\
\text { remorque }\end{array}$ & 15 tonnes \\
\hline $300-400$ & Remorque & $15-75$ tonnes \\
\hline Plus de 400 & Remorque & \\
\hline
\end{tabular}

Source : Nos enquêtes, 2016

La lecture de la figure 1 et le tableau 3 montrent l'usage de plusieurs types de véhicules variables en fonction de la capacité de l'automobile, de l'éloignement du lieu de collecte et de la qualité de la voie qui y mène. Deux éléments d'explication pourraient nourrir la réflexion sur la recrudescence au recours à la marche à pieds et le vélo en amont. Le premier élément se rapporte à la faiblesse de revenu des paysans qui éprouvent des difficultés à s'offrir un moyen de déplacement motorisé. Le deuxième élément d'explication porte sur la faiblesse de la largeur de la route en milieu rural difficilement accessible au gros porteurs. Dans ce cas, ce sont les petits porteurs qui assurent le transport primaire des produits jusqu'au lieu facile d'accès où les gros porteurs prendront le relais. Les résultats trouvés sont identiques à celui d'Aloko (1997) qui confirme que l'automobile demeure en dépit de tout, comme le moyen de transport le plus utilisé. L'usage de la capacité du camion est fonction de l'importance des produits transportés et de l'éloignement de la zone de collecte des produits. Il en est de même du rapport établi par le Ministère du Commerce (1985) qui soutient que les transports à petite distance se font soit par portage, soit sur de petites charrettes à bras, soit en taxis avec un coût très élevé à l'unité de poids. Par ailleurs, les transports à moyenne distance se font habituellement par taxibrousse parce que peu adapté au transport de "colis" de vivriers, surtout périssables.

Au Cameroun, les travaux de Savoye (2000) ont prouvé que les véhicules de petit gabarit prédominent sur les petits et moyens trajets tandis que le transport en longue distance se fait pour une large partie en camions remorque et semi-remorque. Il en est de même pour Razafindramamba (2008) qui affirme que le transport non mécanisé est fréquent car c'est moins cher que les quatre roues à Antanarivo en Madagascar. Dans la même veine, Razafimanadimby (2006) souligne que la subsistance de ce type de moyens de transport traditionnels est due au niveau économique encore plus bas du 
pays. Les moyens traditionnels sont utilisés uniquement pour des quantités limitées de produits transportés. Ceux qui transportent de grandes quantités de produits préfèrent utiliser les moyens mécanisés. Ces moyens sont plus rapides et permettent de faire fonctionner les activités plus vite. Ce résultat reflète bien la réalité de notre espace d'étude contrairement à celui de Baris et Zaslakski (1983) qui mettent en évidence la récurrence des cars en commun dans le transport des produits. Le résultat est en désaccord avec celui de Abdou(2011) qui précise que les animaux de bât (âne, dromadaire et cheval) sont les moyens de transport les plus utilisés au Niger dans la ville de Téra. Car ils permettent de se déplacer à une plus grande vitesse que la marche à pied et de transporter une charge relativement lourde.

\section{Le tarif du transport}

La tarification dépend du trajet de parcours et de la capacité de la charge du camion comme l'indique le tableau ci-dessous.

Tableau 4. Variation du coût de transports imposés par la distance parcourue et selon la capacité de la charge du camion

\begin{tabular}{|c|c|c|c|c|}
\hline $\begin{array}{c}\text { Distance en km } \\
\text { par rapport à } \\
\text { Abidjan }\end{array}$ & Type de véhicule & $\begin{array}{c}\text { Capacité en } \\
\text { tonne }\end{array}$ & $\begin{array}{c}\text { Prix du } \\
\text { transport en } \\
\text { FCFA }\end{array}$ & $\begin{array}{c}\text { Frais de } \\
\text { route/barrage }\end{array}$ \\
\hline $49-125$ & Bâché/frigo nette & 3 & $45000-100000$ & 1000 \\
\hline $200-288$ & $\begin{array}{c}\text { Camionnette } \\
\text { (pickup)/semi- } \\
\text { remorque }\end{array}$ & 5 & 220 à 250000 & 2000 \\
\hline $300-400$ & Remorque & 15 & 300 à 550.000 & 2000 \\
\hline Plus de 400 & Remorque & $15-75$ & 600 à 850000 & 2000 \\
\hline
\end{tabular}

Source : Nos enquêtes, 2016

A l'analyse du tableau, on constate que plusieurs types de véhicules sont utilisés pour le ravitaillement du marché d'Abidjan en produits vivriers en fonction de l'importance ou du volume des produits. Les prix ne sont pas homologués. La variation des prix est non seulement fonction de la capacité de l'automobile, de l'éloignement du lieu de collecte et parfois de la qualité de la voie qui y mène. Les tracasseries routières ainsi que le mauvais état des routes contribuent au coût excessif du prix du transport. La banane plantain, beaucoup plus périssable que les autres produits, est plus vulnérable en cas des conditions de transport difficiles (pannes, mauvais états de routes, etc.) qui peuvent allonger considérablement la durée du voyage.

\section{Les tracasseries routières}

Une étude sur le racket des axes routiers en Côte d'Ivoire réalisée par la Banque Mondiale (2008) reflète bien la réalité de notre espace d'étude. Le 
tableau suivant montre les différents montants relatifs au racket des transports de marchandises.

Tableau 5. Montants du racket relatif à un véhicule de transport de marchandises (camion lourd) observés sur les axes principaux

\begin{tabular}{|c|c|c|c|c|}
\hline Axes & $\begin{array}{c}\text { Nombre } \\
\text { d'observation }\end{array}$ & $\begin{array}{c}\text { Distance } \\
\text { moyenne } \\
\text { parcourue } \\
(\mathrm{km})\end{array}$ & $\begin{array}{c}\text { Montants } \\
\text { moyens du } \\
\text { racket } \\
\text { (FCFA) }\end{array}$ & $\begin{array}{c}\text { Ratios } \\
\text { moyens des } \\
\text { montants du } \\
\text { racket } \\
\text { (FCFA) }\end{array}$ \\
\hline Duékoué-Abidjan & 9 & 456 & 40533 & 8882 \\
\hline $\begin{array}{c}\text { Abidjan- Lareba (frontière du } \\
\text { BurkinaFaso) }\end{array}$ & 24 & 605 & 43138 & 7130 \\
\hline Abidjan-Pogo (Frontière Mali) & 12 & 685 & 35200 & 5139 \\
\hline Abidjan-Bondoukou & 6 & 424 & 52833 & 12461 \\
\hline Abidian-Danané & 4 & 618 & 166500 & 26942 \\
\hline San-Pedro-Abidian & 8 & 328 & 51000 & 15549 \\
\hline Abidjan-Tabou & 8 & 441 & 51000 & 11565 \\
\hline Abidjan-Noé (frontière Ghana) & 12 & 182 & 30500 & 16758 \\
\hline San-Pedro-Daloa & 6 & 262 & 20000 & 7634 \\
\hline
\end{tabular}

Source: Banque Mondiale, 2008

L'analyse de ce tableau montre que les tracasseries routières constituent un véritable casse-tête pour les transporteurs des produits. Les transporteurs sont confrontés à chaque poste aux menaces de fouille des agents des forces de l'ordre chargés du contrôle routier en vertu des droits que leur confère leur profession. Ce sont les agents des Eaux et Forêts, les policiers, les gendarmes, les douaniers, anti-drogue et quelques agents de l'OCPV. Contre cette fouille, il est exigé au transporteur le paiement d'une rançon allant de 30.000 à 100.000FCFA même si tous les camions sont en règle cela ne met pas le transporteur à l'abri des faux frais. Ce phénomène de nature politico-économique trouverait son origine dans l'incapacité du gouvernement à appliquer des sanctions rigoureuses. Cette incapacité a fortement nui à l'efficacité du transport des produits en Côte d'Ivoire. Ces résultats obtenus sont soutenus par Kanga (2013), Yeo (2011) et Kassi (2011) dans les études menées dans le secteur du vivrier. Il en est de même du résultat de Mpanzu (2012) dans son étude menée dans le Bas-Congo en RDC.

\section{Les rugosites au transport des produits vivriers}

Face au transfert des produits, il nous a semblé nécessaire de mettre en relief les obstacles qui s'y opposent. Ceci en tenant compte des rugosités 
qui constituent : " un ensemble de tout ce qui contrarie et freine le transport en s'opposant à sa fluidité »Zambri, $\mathrm{P}$ et al. (2005). Divers composants physiques ou humains génèrent des frictions et des résistances au transport des produits se traduisant à l'allongement de parcours, à l'inaccessibilité, aux tracasseries routières, et a l'insuffisance des infrastructures routières.

\section{L'accessibilité aux zones de productions : une question en suspens}

Le concept d'accessibilité renvoie à deux notions à savoir l'accessibilité économique et l'accessibilité physique.

L'accessibilité économique peut se définir comme la possibilité d'accéder ou de disposer de ressources financières susceptible de satisfaire à un besoin économiques. Elle se caractérise par l'accès à la profession et l'accès aux crédits.

«L'accessibilité physique d'un lieu renvoie la plus ou moins grande facilité par laquelle ce lieu peut-être atteint à partir d'un ou de plusieurs autres lieux, à l'aide de tout ou partie des moyens existants. Elle traduit aussi la pénibilité du déplacement, la difficulté de la mise en relation appréhendée le plus souvent par une fonction décroissante des distances entre les lieux $\gg$. Bavoux et al, (2010). L'accessibilité permet de rendre compte des obstacles à la mise en marché des produits vivriers des zones de production. Dans ce présent article, nous allons analyser le seul angle de l'accessibilité physique. Pour écouler les produits vivriers des lieux de production vers Abidjan, l'accessibilité physique réside au niveau des difficultés à accéder aux champs par les transporteurs. En effet, la création de principales voies de desserte qui date de la période coloniale a suivi un schéma classique appliqué à tous les territoires sous tutelle ou les anciennes colonies allant de la capitale économique (Abidjan) vers l'intérieur du pays pour l'évacuation de matières premières (café cacao, anacarde, coton...). L'accent avait été mis sur les grands axes routiers au détriment de voies intérieures servant à la collecte des produits vivriers. L'entretien du réseau routier a souffert et continue d'ailleurs comme d'autres secteurs de la vie publique, des effets de la crise de ces dernières décennies. Malgré le soutien des partenaires au développement (Banque Mondiale, Fonds Monétaire International, Union Européenne, Banque Africaine de Développement) ces cinq dernières années, l'accent est davantage mis sur le revêtement des grands axes routiers déjà existants.

La majorité des routes sont non asphaltées et difficilement praticables en saison des pluies du fait de l'entretien courant qui n'y se fait pas. Les routes secondaires qui relient certaines zones de productions agricoles sont dégradées. Nous avons des exemples comme la côtière et certaines routes de la ville de Man, comme le montre les photos ci-dessous. D'autres voies telles que Affiénou-Maféré, Tolésso-Maféré dans le département d'Aboisso, 
Bounantafla-Bonon dans le département de Bouaflé qui relient les centres urbains de consommation sont aussi dégradées.

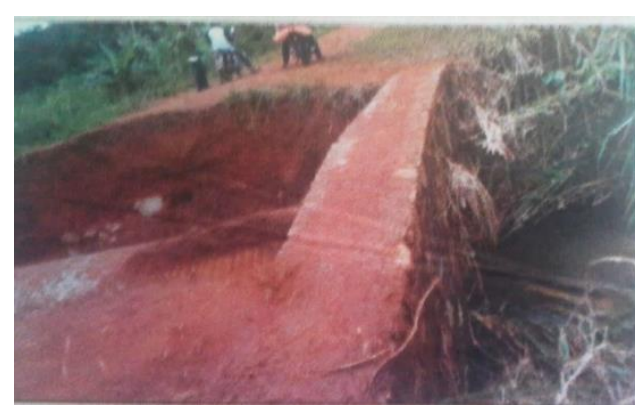

Photo 6. ouvrage endommagé après trois jours de pluies dans la région de Man. Plus de 15 villages sont coupés de la ville. Cliché, Droh h, 2016.

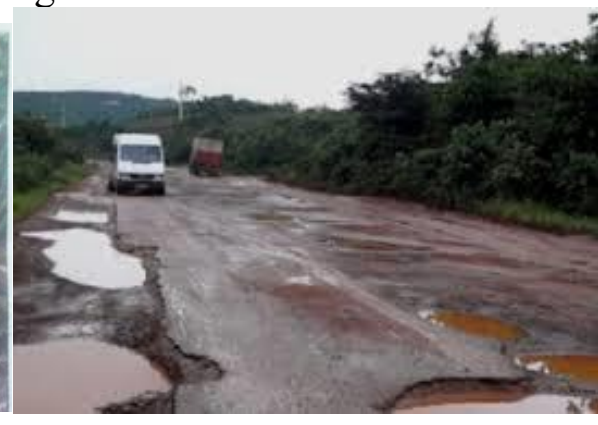

Photo 7. La côtière (Grand-Lahou - San Pedro) fortement dégradée avec des nids d'éléphants constitue un véritable chemin de croix pour les usagers. Cliché : Kouassi F, 2016

L'inégale répartition du réseau de transport routier au niveau nationale et l'étroitesse de la largeur circulable du tronçon Adiaké-AboissoAbidjan qui se voulant économes, occasionnent des charges récurrentes très importantes et se révèlent inadaptées. Outre l'étroitesse handicapante, les routes sont très glissantes en saison pluvieuse et hautement poussiéreuse en saison sèche. Aussi, la présence des trous béants casse les amortisseurs des véhicules. Tous ces lieux «rugueux» contraignent les circulations en allongeant le temps de parcours et suscitent des coûts supplémentaires de carburant, de maintenance aux transports. Le degré d'enclavement justifie aussi l'accès difficile des champs. Il se caractérise par de routes non viable, trop sinueuse couronnée par la pénibilité du trajet à parcourir. Par conséquent les produits pourrissent.

La FAO (1994) estime que ce sont les contraintes organisationnelles, humaines et sociales qui freinent l'approvisionnement alimentaire des villes d'Afrique. L'étude menée par Abdou (2011), révèle que l'insuffisance d'infrastructures modernes de transport et la mauvaise reconversion vers les moyens de déplacement sont des facteurs d'enclavement qu'il qualifie de « rendez-vous manqué avec la modernité ».

Le second facteur explicatif de la faible accessibilité des zones de production sont multiples. L'insuffisance des crédits alloués à la construction, à la réhabilitation et à l'entretien des routes. La trop forte dépendance $\mathrm{du}$ budget d'investissement à l'aide extérieure. Les conditionnalités soumises à l'aide extérieur qui consiste à justifier la rentabilité économique et conséquente d'une route avant de financer sa construction. Selon Abdou (2011), on comprend donc finalement l'idée selon laquelle la route est justifiée par sa fonction économique. 
Dans son étude, Echui (1993) confirme que la faible accessibilité des zones de production rend peu efficace l'organisation de la collecte et de l'évacuation des produits vers les marchés urbains. Au Cameroun, les études de Fofiri et al (2009) ont prouvé que c'est la lourdeur du relief et la faiblesse du réseau de voies de communication qui rendent une région difficile d'accès. Pour lui, le bitumage des axes routiers contribue au désenclavement d'une région sans pourtant résoudre la question de l'accessibilité aux zones rurales. Cette situation est aggravée par le bouleversement climatique.

\section{Le climat préjudiciable aux transports des produits}

La Côte d'Ivoire est le domaine du climat tropical caractérisé par l'alternance de quatre saisons : une grande et une petite saison de pluies, une grande et une petite saison sèche, de durée égale de trois mois chacune. Cependant, la variabilité du climat cause des désagréments au transport des produits vivriers à degré variable en fonction des régions. Ainsi, de Novembre à Janvier, l'harmattan, vent chaud et sec issu des régions des savanes, vient modifier les comportements des transporteurs qui convoient les produits vivriers de Ferkessédougou, Korhogo, Niakara, Dikodougou, Odienné, Bouna, Bondoukou vers Abidjan. En fait, dans ces localités situées au nord et nord-est du pays, ce vent chaud et sec est accompagné d'une brume sèche qui réduit la visibilité des conducteurs. Selon un transporteur, cette situation influence le trajet des transporteurs et est à l'origine de la diminution de la fréquence du transport dans toutes ces localités. En raison du caractère périssable des produits, la rapidité des camions de transport des produits vivriers s'avère indispensable. Or, la brume impose une réduction de la vitesse aux véhicules entrainant des retards fréquents et significatifs. Par conséquent, les produits se détériorent. La fréquence du transport des produits de ces localités vers Abidjan connaissent également une diminution. Avec ces fortes chaleurs dont les températures avoisinent les $40^{\circ} \mathrm{C}$, le mois de mars marque la transition entre la saison sèche et celle des pluies.

Cependant d'Avril en juin, les localités de Bouaflé, San Pédro, Soubré, Yamoussoukro, Aboisso et Man situées au centre-ouest ,sud-est, sud-ouest et ouest du pays connaissent de fortes pluviométries. Ces fortes pluies contribuent à la dégradation prononcée des routes, ralentissent ainsi le rythme de progression du trafic routier des produits. L'humidité complique également le transport des produits vivriers à cause des brouillards qui réduisent la visibilité des chauffeurs provoquant ainsi les ralentissements des véhicules. Les pluies abondantes couplées d'inondations, de glissements de terrain, paralysent le transport des produits vivriers. Par exemple au mois de juin 2016, un ouvrage a été endommagé après trois jours de pluies dans la région de Man. De ce fait, plus de 15 villages ont été coupés de la ville (cf photo 6). L'effondrement du pont du "Corridor de Béréby" (cf photo 8) 
érigé au-dessus du fleuve Diboué à environ $4 \mathrm{~km}$ de San Pedro chef-lieu de la région du Bas Sassandra et l'affaissement du bitume (cf photo 9) de la route de San-Pedro-Abidjan par Gagnoa sous l'effet des abondantes pluies du 10 au 13 juin 2017 sont illustratifs.

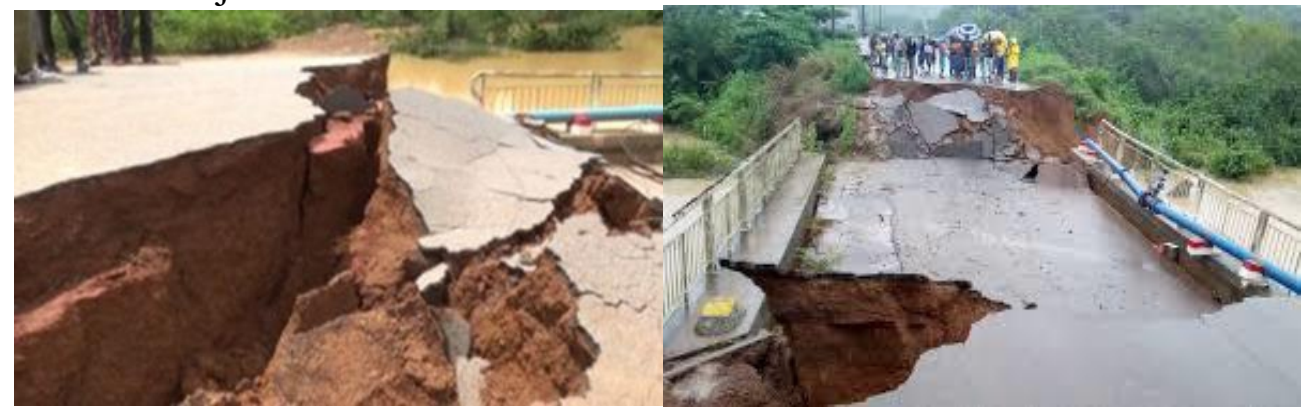

Photo 8. Effondrement du pont du ''Corridor de Béréby" suite aux abondantes pluies dans la région de San Pedro. Cliché : Kouassi, 2017
Photo 9. Route de San-Pedro-Abidjan par Gagnoa coupée où le bitume s'est affaissé après une journée de pluie. Cliché : Kouassi, 2017

Ces résultats corroborent ceux obtenus par Stern et Zehavi (1990) cité par Koetse et Rietveld (2009) au canada qui a montré que le stress lié à la chaleur est un facteur qui modifie le comportement des conducteurs au volant. Il en est de même pour ABDOU (2011) qui conclut que les difficultés du transport résultent des effets anthropiques et géophysiques.

\section{Conclusion}

Le transport joue un rôle capital dans l'approvisionnement d'Abidjan en produits vivriers. Tous les échanges tant nationaux que régionaux dépendent du transport. Bien que la Côte d'Ivoire entière soit en plein chantier, des voies de communications sont créées, le revêtement des chaussées est accentué, le profilage des routes accéléré pour permettre d'avoir un meilleur accès aux produits et de rapprocher ainsi les zones de production, les réseaux et les services de transport sont loin d'être suffisants et demeurent également peu reluisants. L'organisation du transport se fait à trois échelles différentes. L'usage des moyens de transport non mécanisés et mécanisés est fonction du trajet à parcourir et de la qualité des voies d'accès. Le transport se fait par mode terrestre et ferroviaire. Par contre, les transporteurs éprouvent d'énormes difficultés dans l'évacuation des produits vivriers à Abidjan. Il s'agit des contraintes climatiques, de la distance, de la qualité des voies de circulation, de l'insécurité, de l'infection cutanée et de la tracasserie routière. Tous ces maux handicapent sérieusement l'activité des transporteurs, réduisent sensiblement l'efficacité du système et peuvent limiter le transfert de la production d'une région à une autre. 


\section{References:}

1. Abdou Y., (2011,). Transport et désenclavement dans la problématique du développement local à Téra au Niger, Thèse de doctorat, Université de Toulouse, $416 \mathrm{p}$.

2. Aloko J., (1997,). Le ravitaillement de la ville de Bouaké et son impact régional, Cahiers de géographie tropicale, $15 \mathrm{p}$.

3. Banque Mondiale., (2008,). Rapport sur une étude du racket sur les routes en Côte d'Ivoire, 73p.

4. Bavoux et al., (2010,). Géographie des transports, Paris, Armand Colin, $231 \mathrm{P}$.

5. Cruz A., (2011). Transport pour compte propre, un transport routier comme un autre? Pratiques et territoires en France depuis la déréglementation, Thèse de doctorat, Université Cergy- pontoise, 371 p.

6. Djogo., ( 1994, ). Analyse de la politique agricole et alimentaire de la cote d'ivoire. CIRES, Abidjan, 53p

7. Echui., (1993,). Le transport des produits vivriers en Côte d'Ivoire : enjeux et stratégie pour le développement, Thèse ENPC, Paris XH, CERTES, $493 \mathrm{p}$.

8. FAO., http://www.fao.org/waicent/faoinfo/agricult/ags/agsm/sada/sada.htm, Cité le 10 Novembre 2010,

9. Fofiri et al., ( 2011, ). Impact des infrastructures routières dans la structuration et le fonctionnement des espaces marchands au NordCameroun, Dijon, INRA SFER CIRAD, France, $17 \mathrm{p}$.

10. Honagbode., (2010,). Effets des infrastructures de commercialisation sur le commerce des produits vivriers de base au Bénin, CAPOD, 49 p.

11. Kanga M.J., (2013,). Étude géographique des circuits de distribution et de commercialisation des produits vivriers dans l'approvisionnement d'Abidjan, Abidjan, Thèse de doctorat unique, Université Félix Houphouët Boigny de Cocody, 284 p.

12. Kassi, (2011), Transport et Approvisionnement des marchés urbains en produits vivriers,. Côte d'Ivoire, Harmattan, $18 \mathrm{p}$.

13. Koetse et Rietveld, 2009, Stern et Zehavi, (1990,) .cité par Ian Cochran, Infrastructures de transport en France : vulnérabilité au changement climatique et possibilités d'adaptation, rapport CERNA, Mines Paris Tech et Mission Climat de la Caisse des Dépôts. 35 p.

14. Mataski J.L.N., 2006., "Le rôle des goulots d'étranglement de la commercialisation dans l'adoption des innovations agricoles chez les producteurs vivriers du Sud-Kivu (Est de la R.D.Congo)». Dissertation originale présentée en vue de l'obtention du grade de 
docteur en sciences agronomiques et ingénierie biologique Filière : Economie et Développement Rural. p 249.

15. Merenne E., (2013,). Géographie des transports, presses universitaires de Rennes, $16 \mathrm{p}$.

16. Ministère du Commerce, 1985, Rapport sur la commercialisation des produits vivriers en Côte d'Ivoire, Abidjan, $26 \mathrm{p}$.

17. Mpanzu B., (2012,). Commercialisation des produits vivriers paysans dans le Bas-Congo (R D C) : contraintes et stratégies des acteurs. Communauté française de Belgique académie universitaire Wallonie-Europe Université de Liège - Gembloux Agro-Bio Tech, $229 \mathrm{p}$

18. Razafimanadimby A., (2006,). Système de transport routier de marchandises à Madagascar, Mémoire de maitrise, Université d'Antanarivo, $80 \mathrm{p}$.

19. Razafindramamba A., (2008,). Transport urbain à Antanarivo, Mémoire de maitrise,

20. Université d'Antanarivo, $49 \mathrm{p}$.

21. Savoye B., (2000,). Le transport des produits vivriers au Cameroun, pp 33-88 (compléter avec la ville, l'édition, la revue de publication) département des politiques et études division de la macroéconomie et des études.

22. Simmala, (2009,). Transport routier interurbain à KARA au NordTogo, Mémoire de Maitrise, Université de Kara au Togo, 99 p.

23. Yeo L., (2011,). Les femmes dans la commercialisation des produits vivriers à Korhogo : cas du marché à tomate, Abidjan, Mémoire de maîtrise, Université Félix Houphouët Boigny de Cocody-Abidjan, 95 p.

24. Zembri P.et al., (2005,). Réseaux de communication et aménagement du territoire: un état de l'art des approches de la géographie des transports. noisy-le-grand :

25. GDR 903 [Groupement De Recherche 903 «Réseaux »] du CNRS, 1999. $92 \mathrm{P}$

\section{Acronyme}

RGPH : Recensement General de la Population et de l'Habitat

AGEROUTE : Agence de Gestion des Routes

FER : Fonds d'Entretien Routier

OCPV : Office d'aide à la Commercialisation des Produits vivriers

CEA : Commission Economique Africaine 